\title{
Combined use of optical tweezers and scanning electron microscopy to reveal influence of nanoparticles on red blood cells interactions
}

\author{
Tatiana Avsievich ${ }^{*, a}$, Yana Tarakanchikova ${ }^{\mathrm{a}}$, Alexey Popov ${ }^{\mathrm{a}}$, Artashes Karmenyan ${ }^{\mathrm{b}}$, Alexander \\ Bykov $^{\mathrm{a}}$ and Igor Meglinski ${ }^{\mathrm{a}}$ \\ ${ }^{a}$ Optoelectronics and Measurement Techniques, University of Oulu, Oulu, FI-90014, Finland \\ ${ }^{\mathrm{b}}$ Infrared Laboratory, Department of Physics, National Dong Hwa University, Hualien, ROC, \\ Taiwan
}

\begin{abstract}
As a promising drug delivery system, itself or coupled with red blood cells (RBC), nanoparticles (NP) should be studied in frames of their interaction at the cellular level. Experiments were performed on RBC in autologous blood plasma incubated with different $\mathrm{NP}-\mathrm{TiO}_{2}, \mathrm{ZnO}$, nanodiamonds and polymeric nanocapsules. $\mathrm{RBC}$ aggregates formation in RBC suspension was observed with conventional microscopy, while quantitative interaction force measurements between individual RBC was assessed with optical tweezers. Scanning electron microscopy (SEM) imaging demonstrated NP localization and RBC membrane modifications upon binding with NP. Among tested NP, nanodiamonds caused increasing the size of aggregates in $\mathrm{RBC}$ suspensions, $\mathrm{RBC}$ interaction force increase and strong membrane surface modifications, comparing to other tested NP and control sample. Nanocapsules do not cause any adverse effects on RBC properties, confirming biocompatibility and applicability for drug delivery purposes. Optical tweezers combined with SEM imaging serves as fast informative assessment of NP effects on RBC.
\end{abstract}

Keywords: optical trapping, scanning electron microscopy, red blood cells, nanoparticles

\section{INTRODUCTION}

Despite wide use of nanoparticles (NP) in industry and great potential for biomedicine, the lack of knowledge on NP interactions with living systems imposes human health safety concerns. The use of NP in biomedicine proved by a variety of established clinically relevant applications, in particular, drug delivery, imaging nanoprobes and photodynamic therapy agents ${ }^{1}$. Despite the promising use for healthcare, new nanomaterials should overcome practical challenges caused by the lack of understanding on their interaction with living systems ${ }^{2}$. NP, having a large surface area to volume ratio, are very reactive, which can appear as positive and negative regarding to the biological systems. Before the personalized nanodrugs will be introduced into the clinical practice, such aspects of their application as safety limits, efficacy and side effects should be thoroughly studied. Since most of the therapeutic NP inevitably get into the blood flow irrespective of the administration method (oral/intravenous) ${ }^{3}$, elucidation of the mechanisms of NP interaction with blood constituents, as well as their influence on human blood rheological properties is of high importance and urgency.

RBC, being the most abundant blood cell component ( $>99 \%$ ), largely determine blood properties, especially microcirculation, due to the cell mutual interactions governed by RBC aggregation and disaggregation - the reversible clumping of RBC. Variety of methods has been applied to estimate NP toxicity regarding to RBC, such as: optical and transmission electron microscopy ${ }^{4}$, infrared (FTIR) spectroscopy ${ }^{5}$ and fluorescence microscopy ${ }^{6}$. However, they are traditional modalities limiting the number of studied phenomena.

In the present study we estimate changes of RBC interactions with Optical Tweezers (OT) approach and RBC shape modifications, associated with the NP's treatment, by SEM imaging.

*tatiana.avsievich@oulu.fi 


\section{MATERIALS AND METHODS}

\subsection{Red blood cells sample preparation}

Experiments were conducted in autologous blood plasma, obtained from venipuncture blood by two rounds of centrifugation. $\mathrm{RBC}$ were collected prior to experiments by finger pricking method and washed with PBS (pH 7.4) by centrifugation. Obtained RBC were incubated in NP suspension in PBS within 1 hour. Sedimented RBC were accurately taken from the vial and suspended in blood plasma at a concentration of $0.5 \%$ for optical tweezers measurements, and $3 \%$ RBC suspensions for optical microscopy observations. The specimen was placed in a glass sample chamber comprising a glass slide with a coverslip attached with a double-sided adhesive tape to produce a gap of about $100 \mu \mathrm{m}$.

Averages size of tested NP was retrieved from SEM imaging: rutile $\mathrm{TiO}_{2} \mathrm{RODI}$ (Sachtleben, Germany) $-250 \mathrm{~nm}$, alumina-polyol coated anatase $\mathrm{TiO}_{2}$ Hombitan $\mathrm{AN}$ (Kemira, Finland) - $180 \mathrm{~nm}$, uncoated anatase $\mathrm{TiO}_{2} 15 \mathrm{~nm}$ (Oocap, USA), uncoated ZnO (Sigma-Aldrich, Germany) - $270 \mathrm{~nm}$, carboxylated nanodiamonds (Kay Diamond, USA) - 100 $\mathrm{nm}$, and polymeric particles $-600 \mathrm{~nm}$.

\subsection{Optical tweezers}

The OT is a single-cell technique, introduced by Ashkin ${ }^{7}$, enabled dynamic manipulation and precise measurements of interaction forces between individual cells within the $\mathrm{pN}$ range with a focused near-infrared laser beam. Our doublechannel OT setup trapped two RBC simultaneously with Nd:YAG infrared laser beam $(1064 \mathrm{~nm})$ to perform interaction force measurements between them through $\mathrm{RBC}$ aggregation and disaggregation. Measurements were performed in a similar way to our previous studies of mutual interaction of $\mathrm{RBC}^{8,9}$. The aggregation force $\mathrm{F}_{\mathrm{a}}$ of $\mathrm{RBC}$ is the minimum force required to stop two RBC from overlapping. For a single measurement, two $\mathrm{RBC}$ were trapped with individual optical traps, lifted for $30 \mu \mathrm{m}$ and brought in contact at a small overlapping distance of $\Delta \mathrm{x} \sim 2 \mu \mathrm{m}$. From this stable position, the power of one trap starts slowly decreasing until spontaneous aggregation takes place and RBC overlap. The power of the attenuated laser beam registered at this moment is equal to the aggregation force between the two RBC. Disaggregation force is the force applied to separate two overlapped $\mathrm{RBC}$, also was measured to estimate the energy of $\mathrm{RBC}$ interaction.

\subsection{Conventional optical microscopy}

To estimate alterations of RBC aggregation at a multicellular level, light microscopy images were taken using Eclipse LV100DA-U microscope (Nikon, Japan). The samples were prepared in the same cuvettes; RBC were incubated for $2 \mathrm{~h}$ with NP in the same way as described above.

\subsection{Scanning electron microscopy}

For SEM imaging samples were prepared in the same way as for the conventional optical microscopy, and then were fixed in $1 \%$ glutaraldehyde by incubation for $15 \mathrm{~min}$ at room temperature. Samples were accurately washed with with distilled water. A droplet of RBC was dried under vacuum and covered with a 5-nm layer of platinum. SEM imaging was performed with Zeiss Ultra Plus and Sigma field emission scanning electron microscopes (Carl Zeiss, Germany).

\section{RESULTS}

We have measured interaction forces between RBC in blood plasma in presence of different types of NP, including potential candidates as drug carriers. According to conventional microscopy observations, the size of aggregates formed in the sample containing nanodiamonds, was significantly larger, than for other samples, including control.

Among tested NP, nanodiamonds ( $100 \mathrm{~nm}$ ) caused an almost two-fold increase of the aggregation force, while $\mathrm{ZnO}$ (270 $\mathrm{nm})$ and $\mathrm{TiO}_{2}(15,180$ and $250 \mathrm{~nm}) \mathrm{NP}$ did not have change the aggregation of RBC, compared to the control sample, where $F_{a}=4.26 \pm 0.87 \mathrm{pN}$. Corresponding values of the aggregation forces: $F_{a}=7.68 \pm 0.57$ for nanodiamonds, for polymeric nanocapsules $F_{a}=4.87 \pm 1.01 \mathrm{pN}$, for $\mathrm{ZnO} N P F_{a}=4.63 \pm 0.84 \mathrm{pN}$, for $\mathrm{TiO}_{2} 15 \mathrm{~nm} \mathrm{NP} F_{a}=5.15 \pm 0.45 \mathrm{pN}$, for $\mathrm{TiO}_{2}$ Hombitan AN NP $F_{a}=5.23 \pm 0.88 \mathrm{pN}$, for $\mathrm{TiO}_{2}$ RODI NP $F_{a}=5.52 \pm 0.68$. There was no any correlation observed in size of $\mathrm{TiO}_{2} \mathrm{NP}$ and its influence on the $\mathrm{RBC}$ aggregation state.

Disaggregation force measurements demonstrated, that interaction energy between RBC do not change, however in case of nanodiamonds, energy required to fully separate RBC aggregate is higher in comparison to other samples (Fig. 1a). 
Interaction energy presented as a function of interaction energy per conjugated surface area of interacting RBC vs. relative displacement from initial overlapping area $S_{0}$ to the $S_{i}-$ when cells couldn't not be shifted anymore.

OT measurements were complemented with SEM imaging to reveal diversity in NP attachments to RBC and their localization on the membrane together with morphological changes caused by RBC-NP interactions. Again, treatment with nanodiamonds resulted in significant changes of RBC shape (Fig. 1b), while other NP did not cause adverse effects upon interaction.
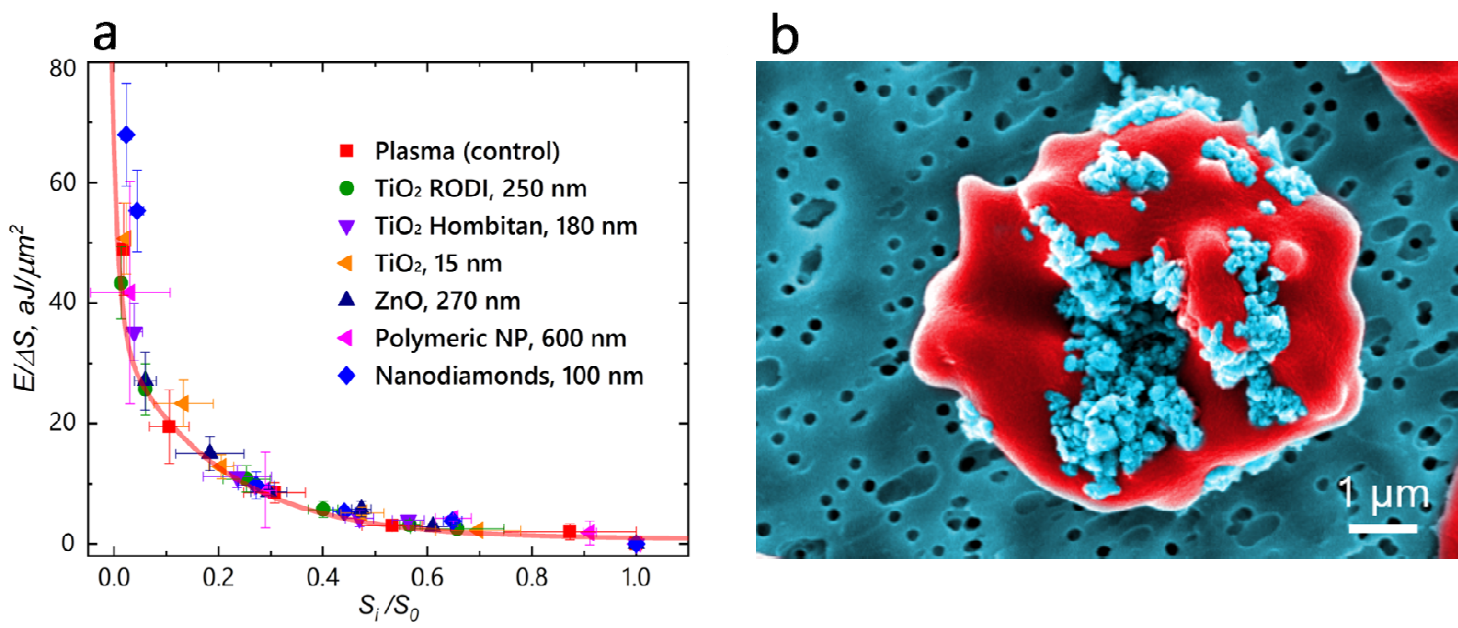

Figure 1. Energy of mutual RBC interaction influenced by NP (a), and Coloured SEM image of nanodiamonds (blue) attached to RBC membrane (b).

\section{CONCLUSIONS}

Presence of nanodiamonds caused morphological changes of RBC membrane and increased RBC interactions forces, indicating a negative impact on $\mathrm{RBC}$ aggregation and the membrane, whereas other NP, including nanocapsules do not change interaction force between $\mathrm{RBC}$ or their shape. Our findings show that nanodiamonds should be significantly modified to be applicable for biomedical purposes. OT serves as a precise method for estimation of RBC response upon NP treatment, and can be used as a first step of NP validation for biomedical use.

\section{REFERENCES}

[1] Salata, O.V. "Applications of nanoparticles in biology and medicine," J. Nanobiotechn. 2(3), 1-6 (2004).

[2] Anderson, D. Sydor, M.J., Fletcher, Holian, P.A. "Nanotechnology: The risks and benefits for medical diagnosis and treatment," J. Nanomed. Nanotechnol. 7:e143 (2016).

[3] Tsai, L.W., Lin, Y.C., Perevedentseva, E., Lugovtsov, A., Priezzhev, A., Cheng, C.L. "Nanodiamonds for medical applications: interaction with blood in vitro and in vivo," Int. J. Mol. Sci. 17(7) (2016).

[4] Chen, L.Q., Fang, L., Ling, J., Ding, C.Z., Kang, B., Huang, C.Z. "Nanotoxicity of silver nanoparticles to red blood cells: size dependent adsorption, uptake, and hemolytic activity," Chem. Res. Toxicol., 501-509 (2015).

[5] Cao, T., Yang, Y., Gao, Y., Zhou, J., Li, Z., Li, F. "High-quality water-soluble and surface-functionalized upconversion nanocrystals as luminescent probes for bioimaging," Biomaterials 2959-2968 (2011).

[6] Park, J.-H., Gu, L., von Maltzahn, G., Ruoslahti, E., Bhatia, S.N., Sailor, M.J. "Biodegradable luminescent porous silicon nanoparticles for in vivo applications," Nature Mater. 331-336 (2009).

[7] Ashkin, A. "Acceleration and trapping of particles by radiation pressure," Phys. Rev. Lett. 156 (1970).

[8] Avsievich, T., Popov, A., Bykov, A., Meglinski, "Mutual interaction of red blood cells assessed by optical tweezers and SEM imaging," Opt. Lett. 43(16), 3921-3924 (2018).

[9] Lee, K., Danilina, A.V., Kinnunen, M., Priezzhev, A.V. and Meglinski, I. "Probing the Red Blood Cells Aggregating Force with Optical Tweezers," IEEE J. Sel. Top. Quantum Electron. 22(3), 7000106 (2016). 